Check for updates

Cite this: RSC Adv., 2020, 10, 25966

Received 15th May 2020

Accepted 3rd July 2020

DOI: $10.1039 / \mathrm{d} 0 \mathrm{ra0} 4348 \mathrm{f}$

rsc.li/rsc-advances

\section{Polyelectrolyte-grafted $\mathrm{Ti}_{3} \mathrm{C}_{2}-\mathrm{MXenes}$ stable in extreme salinity aquatic conditions for remediation of contaminated subsurface environments $\dagger$}

\begin{abstract}
Sehyeong Lim, Hyunsu Park, Jin Hyung Kim, Jeewon Yang, Chaesu Kwak, Jieun Kim, Seoung Young Ryu and Joohyung Lee (iD *

MXenes, an emerging class of two-dimensional materials, are recently gaining significant attention for numerous environmental applications owing to their superior hydrophilicity and unique surface functionalities, which are suitable for adsorptive removal of various aqueous contaminants. However, it has recently been shown that MXenes have poor colloidal stability in both synthetic or natural waters containing small amounts of salt ions, which will limit the potential uses of MXenes in remediation of subsurface environments that might sometimes contain considerable amounts of salt ions, and other relevant environmental applications. Herein, we develop $\mathrm{Ti}_{3} \mathrm{C}_{2}-\mathrm{MXenes}$ grafted with highly salt-resistant polyelectrolytes (PEs), MXene-g-PEs, which are colloidally stable in extreme salinity aquatic environments and have low adsorption to soil mineral substrates. The MXenes grafted with zwitterionic PEs are found to have superior mobility properties to those with anionic PEs, which are attributed to the anti-PE behavior of the grafted polymer brushes. The MXene-g-(zwitterionic) PEs show long-term colloidal stability over 6 months in American Petroleum Institute (API) brine with extreme salinity (ionic strength of $2 \mathrm{M}$ with $\left.182.2 \mathrm{mM} \mathrm{Ca}^{2+}\right)$, and little adsorption $\left(0.5 \mathrm{mg} \mathrm{m}^{-2}\right)$ against $\alpha$-alumina surfaces (2.3 $\mathrm{m}^{2} \mathrm{~g}^{-1}$ ). Furthermore, the MXene- $g$-PEs retained the excellent adsorption capacity for methylene blue as a model aqueous organic pollutant. The results suggest the great potential of the MXene-g-PEs as an aqueous pollutant scavenger for various environmental applications including the combined ex situ/in situ remediation, and other relevant subsurface applications.
\end{abstract}

\section{Introduction}

With the rapid population growth and industrialization, aqueous contaminants originating from various sources, including dwellings, industries, transportation, and oil storage facilities, are an increasingly serious threat to ecosystems and human health. Such contaminants are difficult to manage owing to their persistence, accumulation, and lack of visibility, and enormous costs are generated in their treatment. Therefore, it is very important to secure technologies for effective and economical environmental remediation activities. ${ }^{1}$

Adsorption is one of the most widely used techniques for treatment of aqueous contaminants because it is easy to operate, low-cost, and environmentally friendly. So far, various nanomaterials have been considered as adsorbents, including

Department of Chemical Engineering, Myongji University, 116 Myongji-ro, Cheoin-gu, Yongin, Gyeonggi-do, 17058, Korea.E-mail: ljbro@mju.ac.kr

$\dagger$ Electronic supplementary information (ESI) available: Characterization of the unmodified MXenes, phase behavior of the ungrafted PEs in high salinity environments, further experimental results for the long-term colloidal stability, adsorption on mineral substrates, and dye removal of the MXene- $g$-PEs. See DOI: $10.1039 /$ d0ra04348f activated carbons, ${ }^{2}$ silicas, ${ }^{3,4}$ zeolites, ${ }^{5}$ carbon nanotubes, ${ }^{6}$ and nanoscale zerovalent irons (NZVI). ${ }^{7,8}$ Furthermore, advanced two-dimensional (2D) materials have also been employed in numerous adsorptive water-treatment studies. ${ }^{9-14}$ Among these, transition metal carbides (or "MXenes"), an emerging class of 2D materials, ${ }^{\mathbf{1 2 - 1 4}}$ have recently gained significant attention owing to their remarkable hydrophilicity, high specific surface area, and unique activated surface functionalities which could be highly beneficial for aqueous environmental remediation. In addition, MXenes have distinct advantages of high electronic conductivity, catalytic activity, and even antibacterial properties, integration of which with the fine tunability of their surface chemistry may potentially lead to generation of new types of environmental applications. For example, titanium carbide $\left(\mathrm{Ti}_{3} \mathrm{C}_{2}\right)$-MXenes have shown promising adsorptive performance for various aqueous pollutants including organic dyes (e.g., methylene blue $(\mathrm{MB})),{ }^{15-17}$ toxic metals $\left(e . g\right.$., $\mathrm{Cu}(\mathrm{II}),{ }^{18,19} \mathrm{Cr}(\mathrm{VI}),{ }^{20}$ $\mathrm{Hg}(\mathrm{II})^{21}$ ), and nuclear wastes. ${ }^{22}$ Owing to their $2 \mathrm{D}$ structure, MXenes have also been utilized to fabricate purification membranes, with which aqueous pollutants are successfully sequestrated. ${ }^{23,24}$ Furthermore, MXenes and their engineered derivatives have been employed in diverse environmental applications such as electrochemical separation, ${ }^{25}$ 
photocatalytic degradation of organic pollutants ${ }^{26}$ and development of environmental sensors. ${ }^{27}$

Despite a number of previous studies, the ability of MXenes for scavenging the aquatic pollutants directly from subsurface environments has not been investigated so far. Indeed, various nanomaterials for environmental applications are nowadays being actively considered for in situ remediation where a pollutant scavenger is directly injected into the contaminated subsurface site, which enables active treatment of contamination sources and prevents re-contamination with relatively low costs for its operation. ${ }^{28,29}$

For successful realization of such types of environmental remediation, however, it is of primary importance to secure colloidal stability of the injected pollutant scavenger in an arbitrary subsurface aquatic environment ${ }^{7,8}$ which may contain mono- (e.g. $\left., \mathrm{Na}^{+}, \mathrm{K}^{+}\right)$, di- $\left(\right.$e.g., $\left.\mathrm{Ca}^{2+}, \mathrm{Mg}^{2+}\right)$, and other multivalent salt ions, and sometimes has a very high ionic strength. ${ }^{30,31}$ In high-salinity aquatic media with high ionic strength, in particular, it is very difficult to maintain colloidal stability because of the screening of electrostatic inter-particle repulsion ${ }^{7,8,30-32}$ and complexation of the surface functional groups with di- and multi-valent salt ions, which may cause inter-particle bridging flocculation. ${ }^{33,34} \mathrm{~A}$ recent study directly showed that $\mathrm{Ti}_{3} \mathrm{C}_{2}$-MXenes, the most widely used species among the MXene family, easily precipitated even with minute amounts of multi-valent ions, displaying poor mobility in both synthetic or natural waters. ${ }^{35}$ Thus, it is anticipated that the pristine MXenes, without further engineering, will not definitely be effective in extracting aquatic pollutants from groundwater. Furthermore, the poor mobility of MXenes in high salinity media limits many potentially relevant applications such as electrical resistivity tomography for monitoring subsurface dense non-aqueous phases (DNAPL) source zone, ${ }^{36,37}$ or oil and gas recovery. ${ }^{30-32}$

Herein, we, for the first time, demonstrate the engineered $\mathrm{Ti}_{3} \mathrm{C}_{2}$-MXenes stable in extreme salinity aquatic environments, which would significantly broaden the application range of the MXene family. The key strategy is to chemically graft highly saltresistant polyelectrolytes (PEs), which can remain hydrated even in extreme salinity environments, on to the silanefunctionalized MXene surface. There have been a considerable number of preceding studies on preparation of polymergrafted adsorbents for improving either adsorption capacity ${ }^{38}$ or colloidal stability (or "mobility" as often stated in relevant studies ${ }^{8}$ ), but studies on adsorbents grafted with salt-resistant PEs stable in high salinity media have been relatively much less focused. Obviously, there has been no study on PE grafting of MXenes for improving their colloidal stability in such challenging environments. In this study, the long-term colloidal stability of the PE-grafted MXenes, MXene-g-PEs, are carefully investigated in an extreme salinity environment, the American Petroleum Institute (API) brine (sodium chloride $(\mathrm{NaCl}) 8 \mathrm{wt} \%+$ calcium chloride $\left(\mathrm{CaCl}_{2}\right) 2 \mathrm{wt} \%$; ionic strength of $\sim 2 \mathrm{M}$ and $\left.182.2 \mathrm{mM} \mathrm{Ca}^{2+}\right){ }^{30}$ Furthermore, the resistance of the MXene- $g$ PEs against undesirable adsorption to model mineral substrates in harsh environments is studied. The overall results suggest that the MXenes grafted with the zwitterionic PEs have superior mobility in high salinity media than those grafted with relatively well-known sulfonated anionic PEs, ${ }^{30,39-42}$ which is discussed in terms of the different brush behavior of the grafted PEs in the presence of salt ions. Finally, the ability of the MXene-g-PEs of scavenging a model aqueous organic pollutant, methylene blue, is shown.

\section{Experimental}

\subsection{Materials}

$\mathrm{Ti}_{3} \mathrm{AlC}_{2}$ was purchased from American Elements (USA). LiF was purchased from Waco (Japan). $\mathrm{HCl}, \mathrm{NaCl}, \mathrm{CaCl}_{2} \cdot 2 \mathrm{H}_{2} \mathrm{O}, \mathrm{AA}$, sodium metabisulfite (SM), potassium persulfate (PP), and 1ethyl-3-(3-dimethylaminopropyl)carbodiimide hydrochloride (EDC) were purchased from Daejung Co., Ltd (Korea). TEOS and APTES were purchased from Tokyo Chemical Industry Co., Ltd (Japan). Acetic acid and MB were purchased from Samchun Pure Chemical Co., Ltd (Korea). AMPS, DMAPS, and $\alpha-\mathrm{Al}_{2} \mathrm{O}_{3}$ were purchased from Sigma Aldrich (USA). Lithium chloride (LiCl) and MV were purchased from Junsei Chemical Co., Ltd (Japan). Lithium hydroxide (LiOH) monohydrate was purchased from Duksan Co., Ltd (Korea).

\subsection{Preparation of delaminated $\mathrm{Ti}_{3} \mathrm{C}_{2}$-MXenes}

$0.8 \mathrm{~g} \mathrm{LiF}$ was dissolved in $10 \mathrm{~mL}$ of $9 \mathrm{M}$ aqueous $\mathrm{HCl}$ under magnetic stirring for $5 \mathrm{~min}$, followed by adding $0.5 \mathrm{~g}$ of $\mathrm{Ti}_{3} \mathrm{AlC}_{2}$ - the mixture was stirred at room temperature (RT) for $24 \mathrm{~h}$. The product was copiously washed by DI water via repeated centrifugation and redispersion, until the $\mathrm{pH}$ of the supernatant reached $>6$. In the final step, the product was centrifugated at $3500 \mathrm{rpm}$ for $2 \mathrm{~min}$ to precipitate multilayered MXenes along with residual $\mathrm{Ti}_{3} \mathrm{AlC}_{2}$ and obtain a dark-green aqueous dispersion of delaminated MXenes. The concentration of the asprepared MXene dispersion was measured gravimetrically by evaporating the solvent (typically $2 \mathrm{mg} \mathrm{mL}^{-1}$ ).

\subsection{Synthesis of the PEs}

In the synthesis of poly(AMPS-co-AA) with lower $M_{\mathrm{w}}, 10.3 \mathrm{~g}$ of AMPS, $1.05 \mathrm{~g}$ of AA, $1.62 \mathrm{~g}$ of PP, $1.14 \mathrm{~g}$ of SM, and $60 \mathrm{~g}$ of DI water were stirred at $80{ }^{\circ} \mathrm{C}$ for $16 \mathrm{~h}$, following the procedure reported in the previous literature. In the synthesis of poly(AMPS-co-AA) with higher $M_{\mathrm{w}}, 10.3 \mathrm{~g}$ of AMPS, $1.05 \mathrm{~g}$ of AA, $0.0162 \mathrm{~g}$ of PP, and $60 \mathrm{~g}$ of DI water were used following the same procedure. In the synthesis of poly(DMAPS-co-AA), $10.05 \mathrm{~g}$ of DMAPS, $1.26 \mathrm{~g}$ of AA, $0.036 \mathrm{~g}$ of PP, and $150 \mathrm{~g}$ of DI water were used, following the same procedure.

\subsection{Silane treatment of MXenes}

In the treatment with TEOS, $2 \mathrm{~mL}$ of TEOS was added to $50 \mathrm{~mL}$ of an aqueous MXene dispersion under vigorous stirring, and the $\mathrm{pH}$ was adjusted to 2 with $1 \mathrm{~N} \mathrm{HCl}$, followed by continued stirring at RT for $3 \mathrm{~h}$. The product was washed three times with DI water via repeated centrifugation and redispersion, and the final aqueous dispersion was probe-sonicated in an ice bath for 30 min ( $2 \mathrm{~s}$ operation/1 s pause). In the treatment with APTES, $1 \mathrm{~mL}$ of APTES was preliminarily added to $10 \mathrm{~mL}$ of $5 \mathrm{wt} \%$ acetic 
acid solution, and the mixture was stirred for $15 \mathrm{~min}$, followed by adding $2.5 \mathrm{~N} \mathrm{LiOH}$ to adjust the $\mathrm{pH}$ to 8 . Then, the aqueous dispersion of TEOS-treated MXene at $5 \mathrm{mg} \mathrm{mL}{ }^{-1}$ was added, and the mixture was stirred at $65^{\circ} \mathrm{C}$ for $16 \mathrm{~h}$. The washing and probe-sonication of the product was performed in the same manner as in the previous step.

\subsection{Grafting of PEs to MXenes}

$10 \mathrm{~mL}$ of APTES-treated MXene dispersion (5 $\mathrm{mg} \mathrm{mL}^{-1}, \mathrm{pH}$ ) was added to $20 \mathrm{~mL}$ of $\sim 10 \mathrm{wt} \%$ aqueous PE solution (pH 5 ) of either poly(AMPS-co-AA) or poly(DMAPS-co-AA) under vigorous stirring, and $0.6 \mathrm{~g}$ of EDC was added to the mixture, followed by adjusting the $\mathrm{pH}$ to 5.7 with $\mathrm{LiOH}$ and $\mathrm{HCl}$. After stirring for $15 \mathrm{~min}, 12.5 \mathrm{~mL}$ of $20 \mathrm{wt} \% \mathrm{LiCl}$ solution was added, and the mixture was stirred at RT for $24 \mathrm{~h}$. The washing was performed in the same manner as in the previous steps, and the final dispersion was probe-sonicated in ice bath for $15 \mathrm{~min}(2 \mathrm{~s}$ operation/1 s pause).

\subsection{Batch adsorption test with $\alpha-\mathrm{Al}_{2} \mathrm{O}_{3}$ substrates}

$4 \mathrm{~mL}$ of either DI water or API brine containing MXenes or PEgrafted MXenes (at $2.5 \mathrm{mg} \mathrm{mL}^{-1}$ ) and $4 \mathrm{~g}$ of $\alpha-\mathrm{Al}_{2} \mathrm{O}_{3}$ (average size $\leq 10 \mu \mathrm{m}$, BET surface area of $2.32 \mathrm{~m}^{2} \mathrm{~g}^{-1}$ ) were added in a $20 \mathrm{~mL}$ vial, and stirred with a magnetic stirrer at $300 \mathrm{rpm}$ for $24 \mathrm{~h}$. The stirrer was removed from the vial and the vial was placed on a flat surface to allow $\alpha-\mathrm{Al}_{2} \mathrm{O}_{3}$ particles to settle down for visual inspection of the adsorption of MXenes or PE-grafted MXenes. In the quantitative analysis for the adsorption of MXene- $g$-pol$\mathbf{y}$ (DMAPS-co-AA) onto $\alpha-\mathrm{Al}_{2} \mathrm{O}_{3}$ in API brine, a series of experiments were performed at concentrations of MXene- $g$ poly(DMAPS-co-AA) of $0.1,0.5,1,1.25$, and $2.5 \mathrm{mg} \mathrm{mL}^{-1}$ in $4 \mathrm{~mL}$ API brine with $4 \mathrm{~g}$ of added $\alpha-\mathrm{Al}_{2} \mathrm{O}_{3}$. The equilibrium concentration of the MXene- $g$-poly(DMAPS-co-AA) was estimated by UV-vis spectroscopy based on the calibration curve established for the absorption peak at $320 \mathrm{~nm}$, and the experimental data were fit to the Langmuir isotherm model using a nonlinear least-squares method.

\subsection{Dye removal test}

For the MB removal test in DI water, $5 \mathrm{~mL}$ of DI water containing either MXenes or PE-grafted MXenes was purposely contaminated with $\mathrm{MB}$ at 40, 48, 56, 64, 72, and $80 \mathrm{ppm}$, and was magnetically stirred at $700 \mathrm{rpm}$ for $1 \mathrm{~h}$. The adsorbents were removed by centrifugation at $8000 \mathrm{rpm}$ for $20 \mathrm{~min}$, and the supernatant was collected and syringe-filtered through a nonsterile polyethylene sulfone (PES) membrane with a pore size of $0.2 \mu \mathrm{m}$. The equilibrium MB concentration was then estimated by UV-vis spectroscopy based on the calibration curve established for the absorption peak at $646 \mathrm{~nm}$. For the dye removal test in high-salinity solutions, $5 \mathrm{mg}$ of either MB or MV was added in $50 \mathrm{~mL}$ of aqueous $1 \mathrm{M} \mathrm{NaCl}$ or $\mathrm{CaCl}_{2}$ solutions, and the solution was syringe-filtered through the $0.2 \mu \mathrm{m}$ PES membrane to discard the undissolved dyes owing to the low solubility of the dyes in high-salinity solutions. The typical saturated dye concentration was approximately $15 \mathrm{ppm}$. Then, $6 \mathrm{~mL}$ of $\sim 0.3 \mathrm{M} \mathrm{NaCl}$ or $\mathrm{CaCl}_{2}$ solutions, contaminated either by
MB or MV at $\sim 5$ ppm, was prepared with added PE-grafted MXenes at $3.33 \mathrm{mg} \mathrm{mL}^{-1}$, and stirred at $700 \mathrm{rpm}$ for $1 \mathrm{~h}$. The centrifugation and syringe-filtration were similarly performed to remove the adsorbents and the removal of dyes from the supernatants was evaluated through visual inspection and UVvis spectroscopy. To test the reusability of MXene- $g$-PEs as adsorbents, the adsorbed dyes were washed three times with ethanol and DI water, respectively, using centrifugation. The dye removal test was then performed under identical conditions using the washed MXene- $g$-PEs. The washing and removal test were repeated up to five times.

\subsection{Characterization}

Scanning electron microscopy (SEM, S-4800, Hitachi, Japan), Xray diffractometry (XRD, AERIS, Malvern, UK), and Fourier transform infrared spectroscopy (FTIR, Varian 660-IR, VARIAN, USA) were used to characterize the morphology, crystalline properties, and chemical functional groups of the delaminated MXenes. X-ray photoelectron spectroscopy (XPS, Thermo Scientific K-Alpha ${ }^{+}$, Thermo Fischer Scientific, USA) was used to perform chemical analyses of products obtained after each reaction step to evidence the success of surface modification. Transmission electron microscopy (TEM, JEM-2100F, JEOL, Japan) was used to characterize the morphology of the PEgrafted MXenes. Dynamic light scattering (DLS) and phase analysis light scattering (PALS) were performed to measure the hydrodynamic diameter $\left(d_{\mathrm{H}}\right)$ and zeta potential of the particles, respectively, using a Zetasizer ZS90 (Malvern, UK). GPC (EcoSEC HLC-8320, Tosoh, Japan) was performed to characterize the $M_{\mathrm{w}}$ of the synthesized PEs. TGA (SDTA851, Mettler Toledo, USA) was used to estimate the amounts of grafted PEs. UV-vis spectroscopy (S-3100, SCINCO, Korea) was used to characterize the particle and dye concentration.

\section{Results and discussion}

\subsection{Development of the PE-grafted MXenes}

We prepared delaminated $\mathrm{Ti}_{3} \mathrm{C}_{2}$-MXenes by etching titanium aluminum carbide $\left(\mathrm{Ti}_{3} \mathrm{AlC}_{2}\right)$ with a high-concentration aqueous hydrogen chloride ( $\mathrm{HCl}, 9 \mathrm{M}$ ) and excess lithium fluoride ( $\mathrm{LiF}$, $0.8 \mathrm{~g}$ per $0.5 \mathrm{~g}$ of $\mathrm{Ti}_{3} \mathrm{AlC}_{2}$ ), following a recently reported procedure. $^{43}$ The as-prepared delaminated MXenes had excellent water dispersibility owing to the presence of hydrophilic hydroxyl surface functional groups, which are inferred from the significant absorption peaks at $3340 \mathrm{~cm}^{-1}$ and $1639 \mathrm{~cm}^{-1}$ of the FTIR spectra (Fig. S1 $\dagger$ ), and electrostatic particle repulsion, which is inferred from the measured high zeta-potential (Fig. 1).

Two types of PEs were prepared to be grafted onto the MXene surfaces. The first PE is an anionic random copolymer of 2acrylamido-2-methylpropane sulfonic acid (AMPS) and acrylic acid (AA), poly(AMPS-co-AA), where AMPS is a stabilizing unit for colloidal stability in high-salinity conditions and AA is an anchoring unit supposed to condense on the MXene surface (Fig. 1a) - this anionic PE has been widely employed in a number of studies relevant to subsurface oil and gas applications. $^{30,39-42}$ The second $\mathrm{PE}$ is a zwitterionic random 
(a)

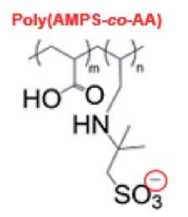

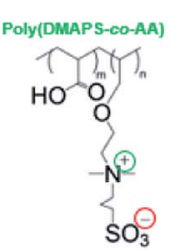

(b)

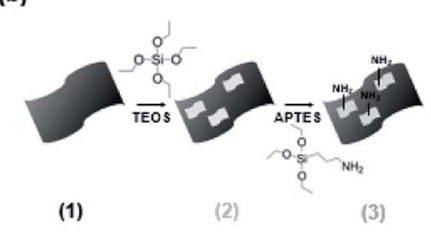

(4)-Anionic

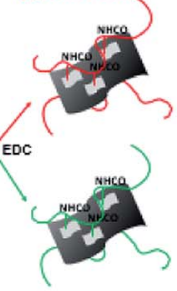

(c)

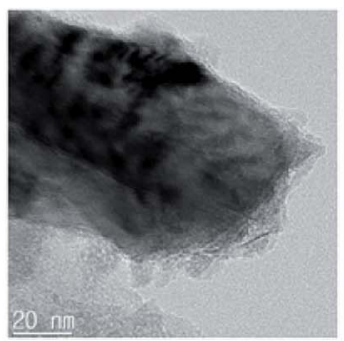

(d)

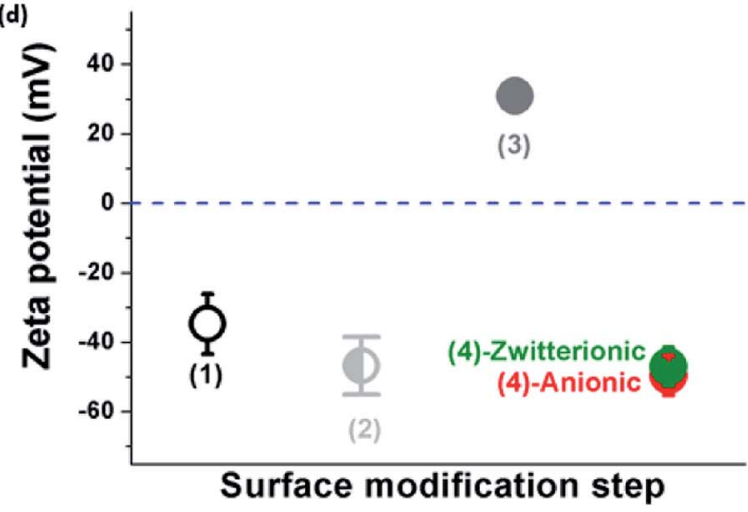

(4)-Zwitterionic

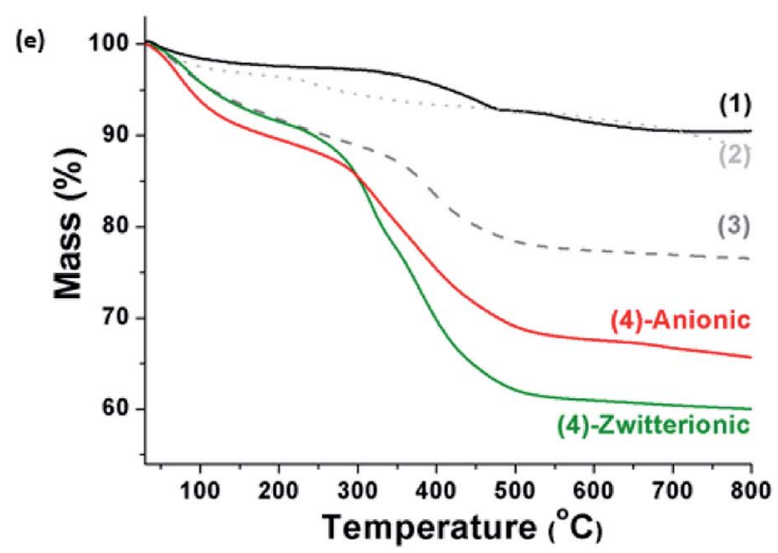

Fig. 1 (a) Chemical structures of the synthesized copolymers. (b) Schematic diagram of the covalent PE grafting onto silane-treated MXenes. (c) Representative TEM image of the MXene-g-poly(DMAPS-co-AA). (d) Zeta potential and (e) TGA analysis at each step of the surface modification procedure.

copolymer of [2-(methacryloyloxy)ethyl]dimethyl-(3-sulfopropyl) ammonium hydroxide (DMAPS) and AA, poly(DMAPS-co-AA), where DMAPS is a stabilizing unit and AA is an anchoring unit (Fig. 1a). The as-synthesized PEs were characterized with gel permeation chromatography (GPC) and the weight-average molecular weight $\left(M_{\mathrm{w}}\right)$ is presented in Table 1.

To achieve covalent grafting of PEs, the MXenes were treated with tetraethyl orthosilicate (TEOS), and subsequently with (3aminopropyl)triethoxysilane (APTES) ${ }^{30,44}$ The pre-treatment with TEOS was expected to form siloxanes covalently bonded to the oxygen-bearing surface sites of the MXenes, ${ }^{44}$ facilitating the condensation of APTES molecules in the siloxane-to-surface direction rather than the electrostatic binding of APTES in the amine-to-surface direction which would be disadvantageous for anchoring of the PEs. After treatment with TEOS, the XPS spectra obtained for the product showed a characteristic Si $2 \mathrm{p}$ photoelectron peak at a binding energy of $103.4 \mathrm{eV}$ (Fig. $2 \mathrm{~b}$ and 3a) which was not observed in the untreated MXenes (Fig. 2a and $3 \mathrm{a})$. In addition, a significant shift in the $\mathrm{O} 1 \mathrm{~s}$ peak to

Table 1 Weight-average molecular weight $\left(M_{w}\right)$ of the as-synthesized PEs

\begin{tabular}{ll}
\hline Polyelectrolyte & $M_{\mathrm{w}}$ \\
\hline Poly(AMPS-co-AA) & $77024 \mathrm{~g} \mathrm{~mol}^{-1}$ \\
Poly(DMAPS-co-AA) & $123823 \mathrm{~g} \mathrm{~mol}^{-1}$
\end{tabular}

higher binding energy was observed (Fig. 3b), suggesting the condensation of silane molecules onto oxygen-bearing surface sites of the MXenes. ${ }^{44,45}$ After treatment with APTES, a distinct $\mathrm{N}$ 1s peak was observed at $401.64 \mathrm{eV}$, which was attributed to the amine group of APTES (Fig. 2c and 3c). Furthermore, the sign for the zeta potential of MXenes was inverted from negative to positive (Fig. 1d), which confirms that the amine surface functional groups were successfully implanted on the MXene surface. Then, a multi-point covalent grafting ${ }^{41}$ of the AA groups of the two PEs onto the amine groups of the MXene surface was achieved through the economical carbodiimide coupling chemistry, as represented in Fig. 1b, resulting in the MXene-gpoly(AMPS-co-AA) and MXene- $g$-poly(DMAPS-co-AA). A representative TEM image (Fig. 1c) indicates that the product maintained the flake-like structure. The XPS spectra showed characteristic S 2p peaks at $167.83 \mathrm{eV}$ for MXene- $g$-poly(AMPSco-AA) (Fig. 2d and 3d) and at $166.94 \mathrm{eV}$ for MXene- $g$-poly(DMAPS-co-AA) (Fig. 2e and 3d), respectively, indicating the presence of sulfur element, which likely originated from the grafted PEs, in both products. Also, two distinct N 1s peaks were observed for both products, where the peaks of lower binding energies, i.e., $398.32 \mathrm{eV}$ for MXene-g-poly(AMPS-co-AA) and $397.86 \mathrm{eV}$ for MXene- $g$-poly(DMAPS-co-AA) (Fig. 3c), might indicate the formation of amide bond ${ }^{46}$ between the carboxyl anchoring groups of PEs and amine groups on silane-treated MXene surfaces. As presented in Fig. 1d, the sign for the zeta potential of MXene- $g$-PEs was inverted from positive back to negative, suggesting successful PE coating on the aminated 

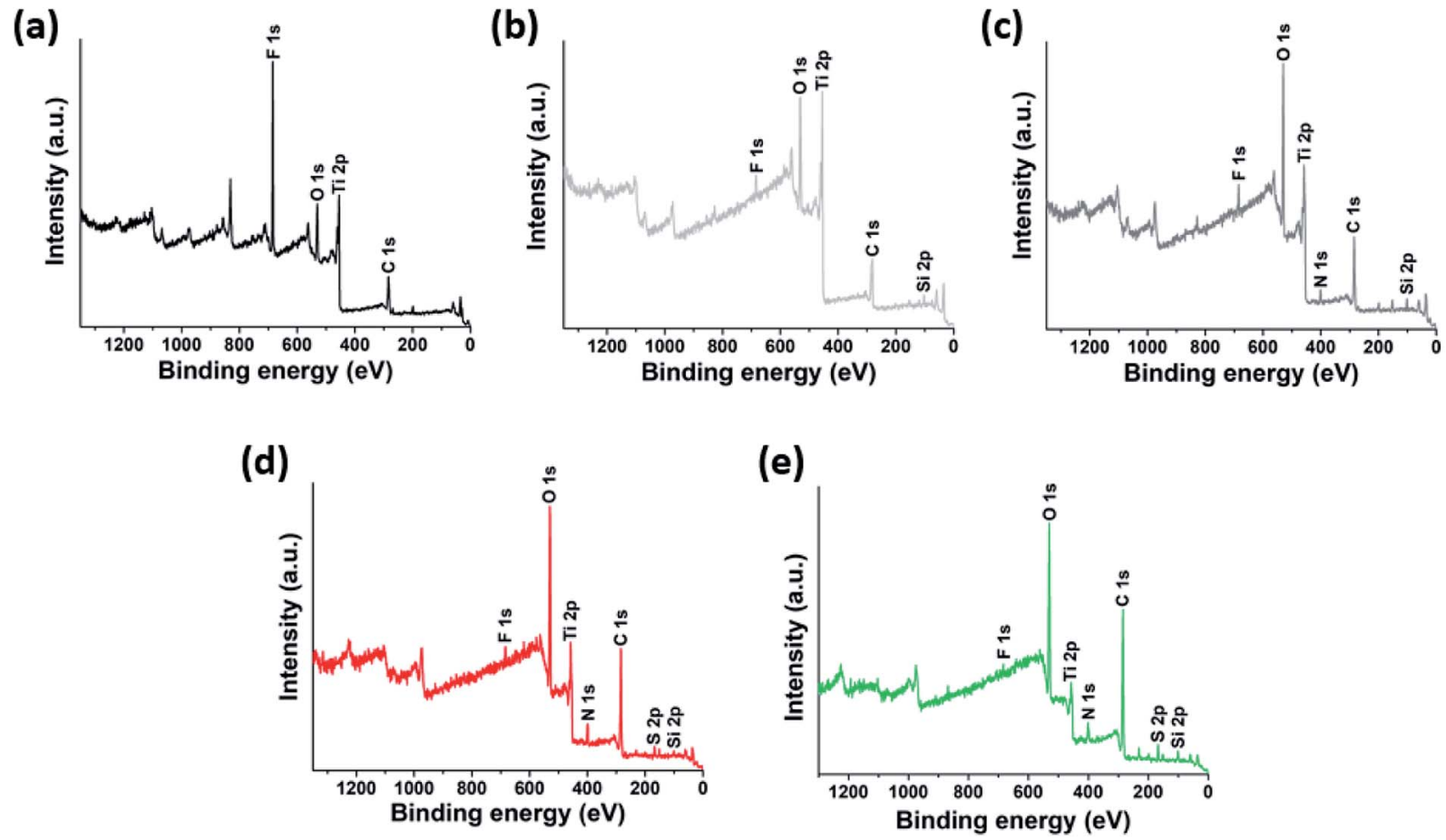

Fig. 2 XPS survey spectra of the products obtained at each surface modification step: (a) MXene ((1)), (b) TEOS-treated MXene ((2)), (c) APTEStreated MXene ((3)), (d) MXene-g-poly(AMPS-co-AA) ((4)-anionic), (e) MXene-g-poly(DMAPS-co-AA) ((4)-zwitterionic).

MXenes. Thermogravimetric analysis (TGA, Fig. 1e) indicates PE contents of $\sim 14.15 \mathrm{wt} \%$ for MXene- $g$-poly(AMPS-co-AA) and $\sim 21.58 \mathrm{wt} \%$ for MXene- $g$-poly(DMAPS-co-AA). The ratio of the grafted mass of poly(DMAPS-co-AA) per gram of MXene ( $m_{\text {po- }}$ ly(DMAPS-co-AA) $/ m_{\text {MXene) }}$ relative to the grafted mass of poly(AMPSco-AA) per gram of MXene ( $\left.m_{\text {poly(AMPS-co-AA) }} / m_{\text {MXene }}\right)$, i.e., $m_{\text {po- }}$ ly(DMAPS-co-AA) $/ m_{\text {poly(AMPS-co-AA) }} \sim 1.67$, is approximately consistent with the ratio of the $M_{\mathrm{w}}$ of the poly(DMAPS-co-AA) to that of the poly(AMPS-co-AA), i.e., $\quad M_{\mathrm{w}, \text { poly(DMAPS-co-AA) }} / M_{\mathrm{w}, \text { poly(AMPS-co-AA) }}$ $\sim 1.6$, indicating that a similar number of PE chains were grafted on the MXene surface.

\subsection{Colloidal stability in the extreme salinity condition}

Fig. 4a shows the dispersion state of the unmodified pristine MXenes and the MXene-g-poly(AMPS-co-AA) in the extremesalinity API brine (sodium chloride $(\mathrm{NaCl}) 8 \mathrm{wt} \%+$ calcium chloride $\left.\left(\mathrm{CaCl}_{2}\right) 2 \mathrm{wt} \%\right)^{30}$ While the pristine MXenes aggregated, formed macroscopic clumps, and precipitated immediately in the API brine, the MXene-g-poly(AMPS-co-AA) displayed excellent dispersion with no visible aggregation, which is attributed to the steric effects caused by the grafted PE chains. However, the PE-grafted MXenes also precipitated under a gravitational field within a few days (Fig. 4 b) because they had a hydrodynamic diameter $\left(d_{\mathrm{H}}\right)$ as large as a few microns (Fig. 4c). Although the precipitation of MXene-g-PEs can be readily restored by simple handshaking, and thus, it is characteristically different from the rapid precipitation of the pristine MXenes caused by their irreversible aggregation, it may still be undesirable for achieving high mobility in the subsurface field application. To overcome this problem, we probesonicated the MXene- $g$-PEs and reduced the $d_{\mathrm{H}}$ down to the submicron level (Fig. 4c). As a result, the sedimentation rate was significantly lowered, in such manner that no evidence of macroscopic phase separation was observed by the naked eye for a few weeks (Fig. 4b). Although the larger size MXene flakes are commonly considered superior for other types of applications (e.g., electronics), it is anticipated that the pulverized nanosized MXenes, with the grafted PEs, would be more suitable for subsurface applications given its higher colloidal stability. Similar pulverization effects were also found for the MXene- $g$-poly(DMAPS-co-AA), as represented in Fig. S2. $\dagger$

Fig. 5a compares long-term colloidal stability of the probesonicated MXene- $g$-poly(AMPS-co-AA) $\left(d_{\mathrm{H}}=214.4 \pm 7.8 \mathrm{~nm}\right.$ at $t=0)$ and the MXene- $g$-poly(DMAPS-co-AA) $\left(d_{\mathrm{H}}=234.0 \pm\right.$ $6.9 \mathrm{~nm}$ at $t=0$ ) observed over the course of two months. Although both particles were colloidally stable for more than a month, a visible phase separation occurred for the MXene-gpoly(AMPS-co-AA) at day 38 which continued to result in complete sedimentation after approximately 2 months. On the other hand, the MXene- $g$-poly(DMAPS-co-AA) showed no visible sedimentation for more than 6 months (Fig. S3†). The difference in the sedimentation stability for both particles may be attributed to the difference in the behavior of the grafted anionic and zwitterionic PE chains in the high-salinity aquatic environment. Typically, PEs with identical sign for the chargeable unit, either polyanions or polycations, tend to contract with increasing ionic strength of the solvent due to the charge screening effects and the decrease in the osmotic pressure caused by the counterions. ${ }^{32,47-49}$ Furthermore, di- or multi- 
(a)

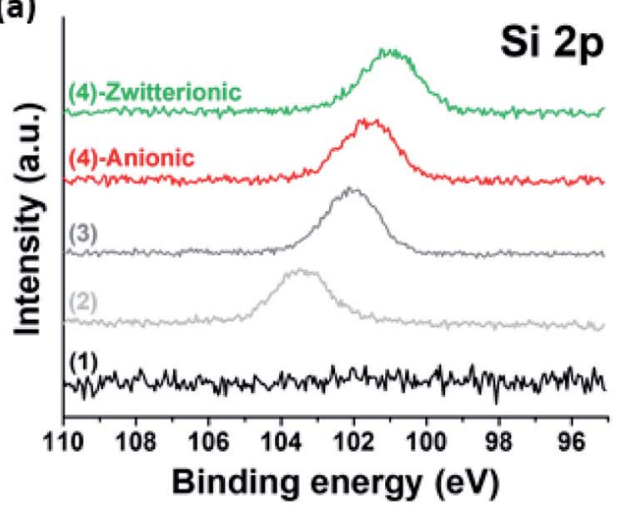

(c)

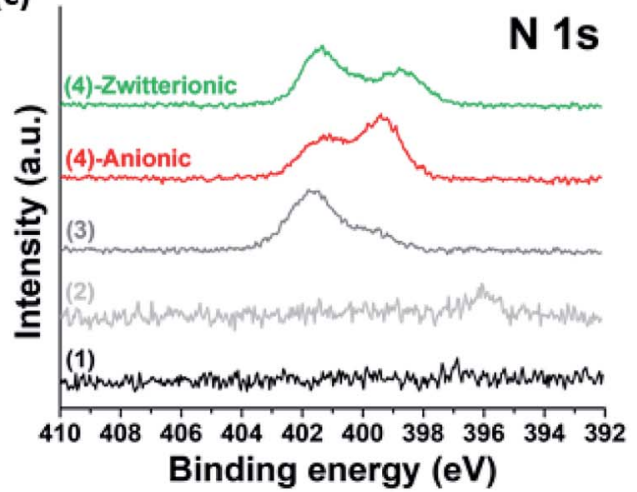

(b)

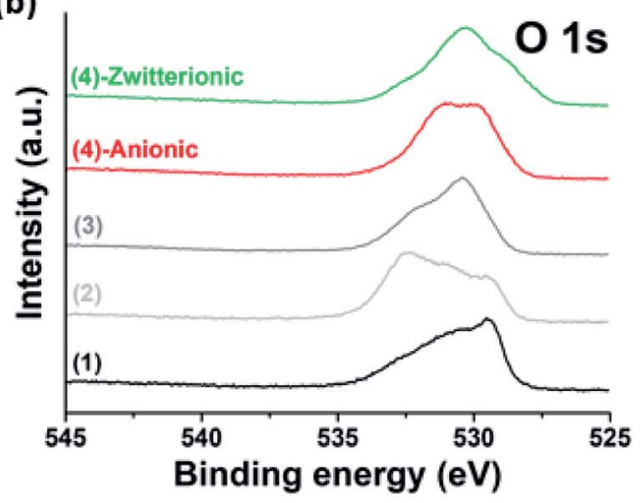

(d)

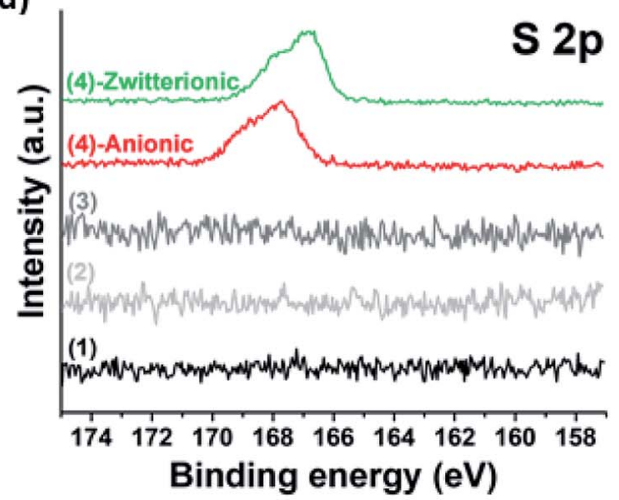

Fig. 3 XPS narrow scan spectra for (a) Si 2p, (b) O 1s, (c) N 1s, and (d) S 2p of the products obtained at each surface modification step: MXene ((1), black), TEOS-treated MXene ((2), light grey), APTES-treated MXene ((3), grey), MXene-g-poly(AMPS-co-AA) ((4)-anionic, red), and MXene- $g$ poly(DMAPS-co-AA) ((4)-zwitterionic, green).

valent counterions undergo complexation with such PEs, causing dehydration and further contraction of the PEs. ${ }^{32-34,50}$ Some anionic PEs consisting of "weak" acid groups, such as poly(AA), have high binding affinity for divalent counterions, and cannot act as an effective steric colloidal stabilizer in the high-salinity environments relevant to subsurface applications (e.g., API brine). ${ }^{51-53}$ In contrast, AMPS has a "strong" acid sulfonic $\left(\mathrm{SO}_{3}{ }^{-}\right)$group which is ionized in the entire $\mathrm{pH}$ range and has much lower binding affinity for divalent counterions. $^{32,54}$ The complexation with divalent counterions is further prevented thermodynamically by the presence of the hydrophilic amide group. ${ }^{55,56}$ Because of this, PEs containing the AMPS unit have been widely employed for various subsurface oil and gas applications in high-salinity environments. ${ }^{30,39-42}$ (a)

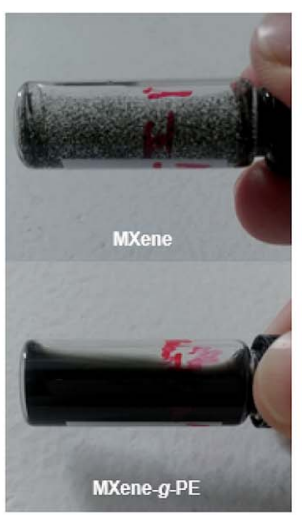

(b)

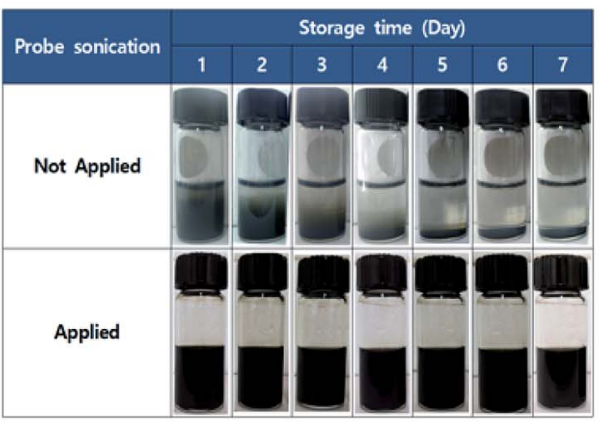

(c)

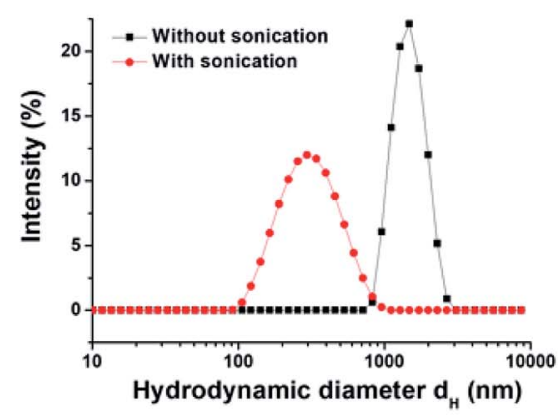

Fig. 4 (a) Dispersion state of the pristine MXenes (upper) and the MXene-g-poly(AMPS-co-AA) (lower) in API brine. (b) MXene-g-poly(AMPS-coAA) in API brine, prepared with (upper) or without (lower) applied probe-sonication. (c) Hydrodynamic diameter distribution of the MXene- $g$ poly(AMPS-co-AA) prepared with (red) or without (black) applied probe-sonication. 
(a)

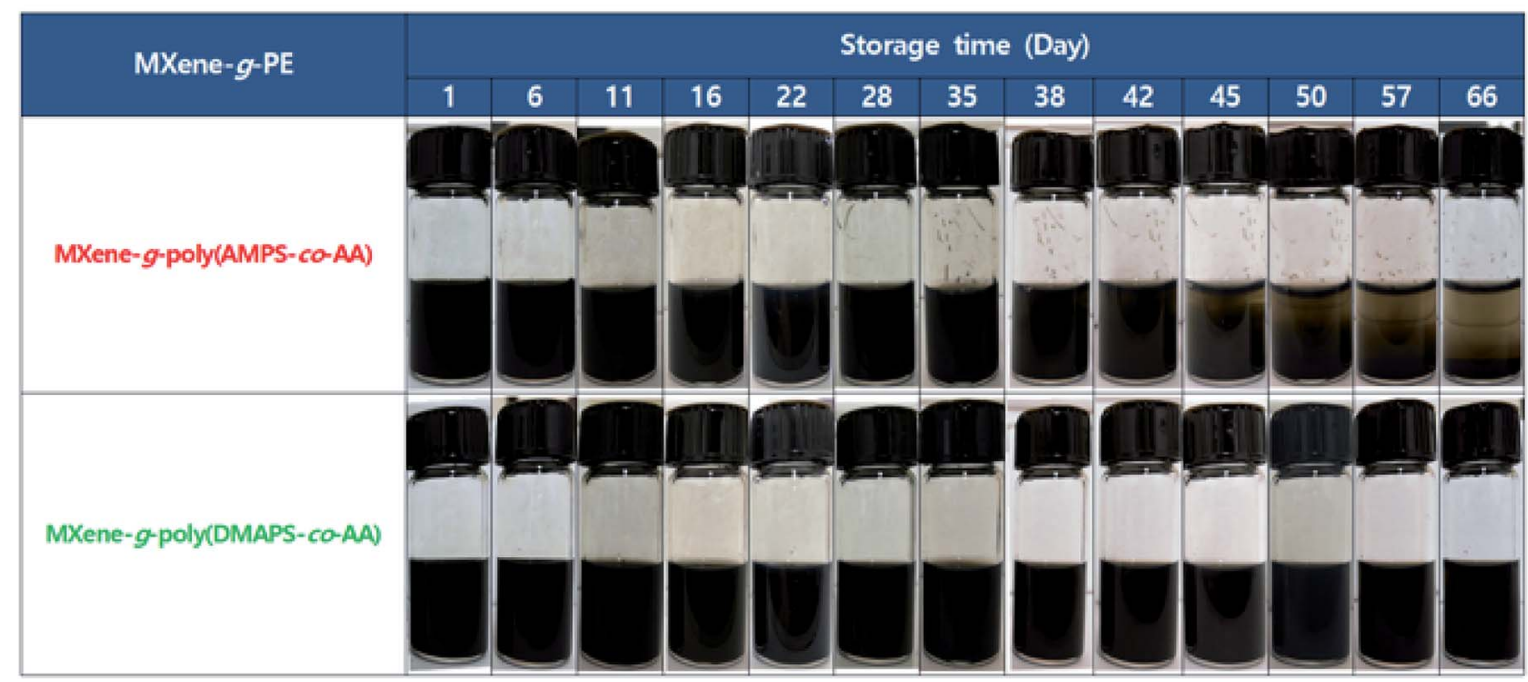

(b)

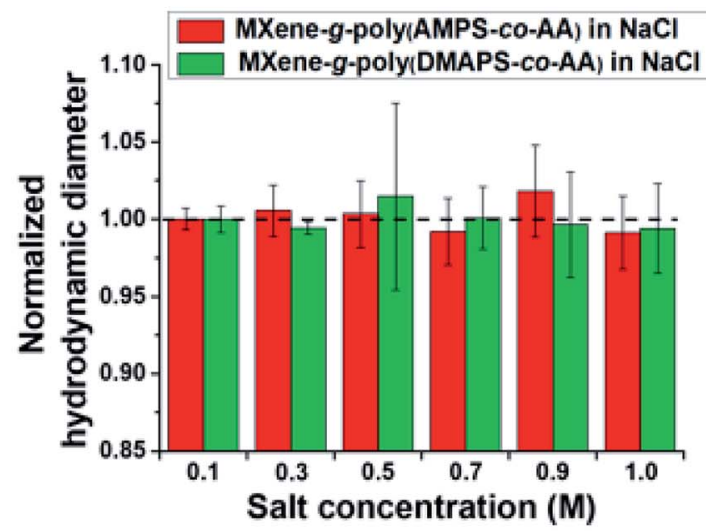

(c)

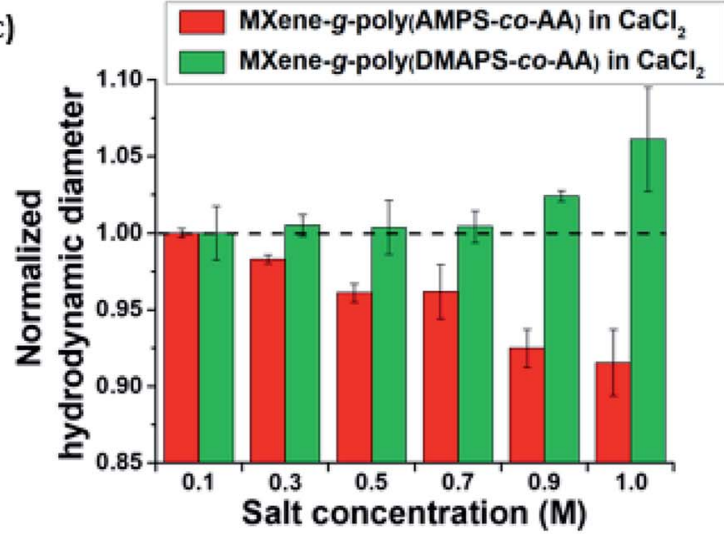

Fig. 5 (a) Comparison of the long-term colloidal stability of the MXene-g-poly(AMPS-co-AA) (upper) and MXene-g-poly(DMAPS-co-AA) (lower) in an API brine. The $d_{H}$ of the MXene- $g$-poly(AMPS-co-AA) and MXene- $g$-poly(DMAPS-co-AA) within $0.1 M \leq C_{s} \leq 1.0 \mathrm{M}$, normalized by the $d_{H}$ at $C_{\mathrm{s}}=0.1 \mathrm{M}$ in the (b) $\mathrm{NaCl}$ and (c) $\mathrm{CaCl}_{2}$ solutions.

However, this does not mean that the AMPS unit is perfectly free from any specific complexation with divalent counterions. For example, ${ }^{32}$ it was recently shown that monodisperse poly(AMPS) brushes end-grafted on silica nanoparticles underwent charge inversion by $\mathrm{Ca}^{2+}$ binding with excess $\mathrm{Ca}^{2+}$ and even the temperature-dependent "second brush collapse," which describes the significant brush re-contraction that occurred after the brushes preliminarily underwent the "first brush collapse" regime caused by the charge neutralization at relatively lower $\mathrm{Ca}^{2+}$ concentrations. These findings strongly (a)

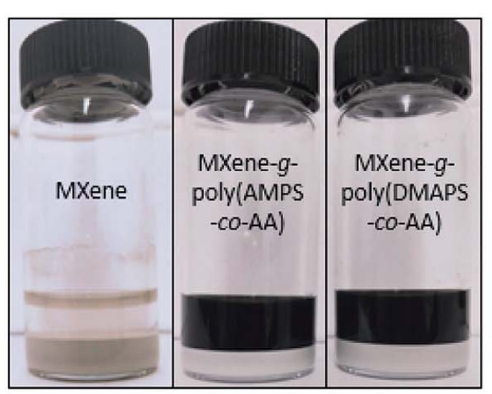

(b)

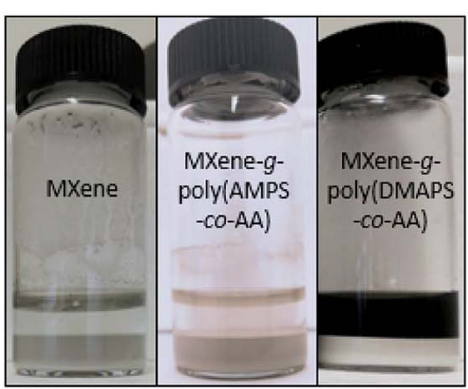

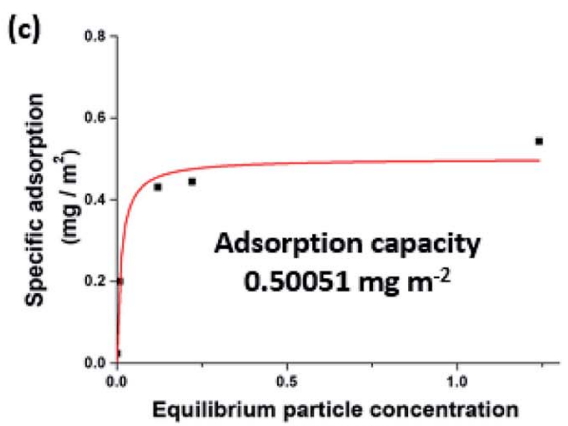

$(\mathrm{mg} / \mathrm{ml})$

Fig. 6 Adsorption of the pristine MXene, MXene- $g$-poly(AMPS-co-AA), and MXene- $g$-poly(DMAPS-co-AA) (from left to right) onto $4 \mathrm{~g}$ of $\leq 10$ $\mu \mathrm{m} \alpha-\mathrm{Al}_{2} \mathrm{O}_{3}$ (BET surface area of $2.32 \mathrm{~m}^{2} \mathrm{~g}^{-1}$ ) in (a) DI water and (b) API brine. (c) Quantitative analysis for adsorption of the MXene-g-poly(DMAPS-Co-AA) onto $\alpha-\mathrm{Al}_{2} \mathrm{O}_{3}$ in API brine based on Langmuir isotherm model. 
(a)

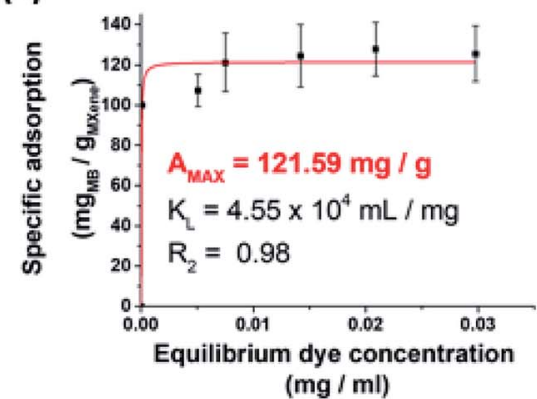

(b)

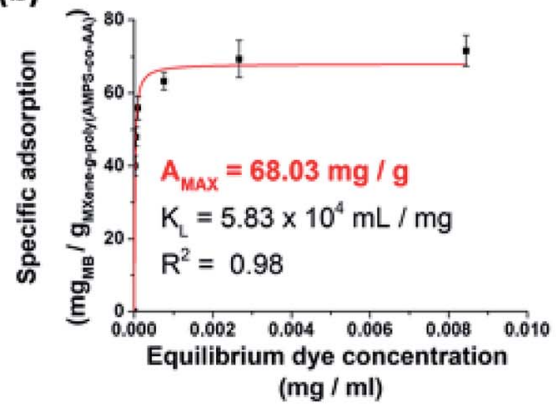

(c)

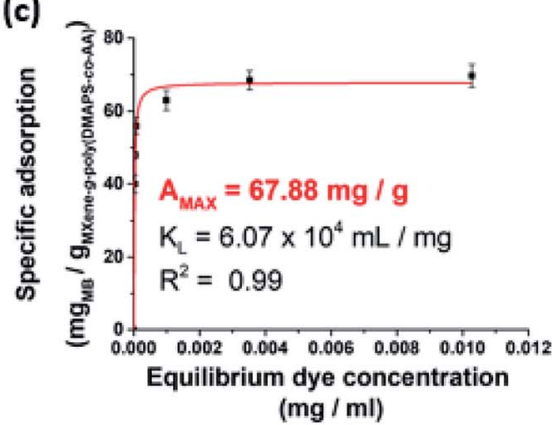

Fig. 7 Quantitative analysis for adsorption of MB onto (a) the pristine MXene, (b) MXene-g-poly(AMPS-co-AA), and (c) MXene-g-poly(DMAPSco-AA) in DI water.

suggested the possibility of specific interaction between poly(AMPS) and $\mathrm{Ca}^{2+}$ ions. In contrast, the zwitterionic PEs could osmotically swell at increased ionic strength accompanying the screening of the intra- and/or inter-chain electrostatic attraction between the oppositely charged PE groups, which could increase the positive excluded volume effects of the PEs - this is referred to as the "anti-polyelectrolyte" effect. ${ }^{31}$ Fig. S4 $\uparrow$ shows the phase behavior of ungrafted poly(AMPS-co-AA) and poly(DMAPS-co-AA) in $\mathrm{NaCl}$ and $\mathrm{CaCl}_{2}$ solutions at high salt concentrations $\left(C_{\mathrm{s}}\right)$, at $C_{\mathrm{s}}=1.0 \mathrm{M}$ and $4.0 \mathrm{M}$. In the $\mathrm{NaCl}$ solutions, both polymers were soluble up to $C_{\mathrm{s}}=4.0 \mathrm{M}$. In the $\mathrm{CaCl}_{2}$ solutions, however, poly(AMPS-co-AA) showed poor solubility at $C_{\mathrm{s}}=4.0 \mathrm{M}$, whereas poly(DMAPS-co-AA) remained soluble, indicating that the zwitterionic poly(DMAPS-co-AA) had higher resistance to the divalent $\mathrm{Ca}^{2+}$. Fig. $5 \mathrm{~b}$ and $\mathrm{c}$ show the $d_{\mathrm{H}}$ of the MXene-g-poly(AMPS-co-AA) and the MXene-g-poly(DMAPS-co-AA) in $\mathrm{NaCl}$ and $\mathrm{CaCl}_{2}$ solutions within $0.1 \mathrm{M} \leq C_{\mathrm{s}} \leq$ $1.0 \mathrm{M}$, which was normalized by the $d_{\mathrm{H}}$ at $C_{\mathrm{s}}=0.1 \mathrm{M}$. In the $\mathrm{NaCl}$ solutions (Fig. 5b), no significant variation in the measured $d_{\mathrm{H}}$ was observed with increasing $C_{\mathrm{s}}$, suggesting that the grafted brushes maintained their hydrated state at $C_{\mathrm{s}}=$ $0.1 \mathrm{M}$. In the $\mathrm{CaCl}_{2}$ solutions (Fig. $5 \mathrm{c}$ ), however, the $d_{\mathrm{H}}$ of the MXene- $g$-poly(AMPS-co-AA) decreased gradually with the increasing $C_{\mathrm{s}}$, which reflected the contraction of the grafted poly(AMPS-co-AA) brushes, whereas the $d_{\mathrm{H}}$ of the MXene-gpoly(DMAPS-co-AA) increased, which is attributed to the swelling of the grafted poly(DMAPS-co-AA) brushes. Thus, the results suggest the importance of specific $\mathrm{PE}-\mathrm{Ca}^{2+}$ interaction for the sedimentation stability of the PE-grafted MXenes in the API brine. The grafted poly(AMPS-co-AA) chains, which contracted with increasing $\mathrm{Ca}^{2+}$, likely formed a more compact brush layer with higher effective density than the poly(DMAPSco-AA) chains, which swelled and had a higher excluded volume with increasing $\mathrm{Ca}^{2+}$. This explains the relatively faster sedimentation of the MXene- $g$-poly(AMPS-co-AA) than the MXene- $g$ poly(DMAPS-co-AA) under the gravitational field.

One may argue that the difference in the sedimentation stability shown in Fig. 5a might simply be caused by the difference in the $M_{\mathrm{w}}$ of both polymers, not by the difference in the mechanistic behavior (i.e., swelling - contraction) of the grafted anionic and zwitterionic PEs in high-salinity environments. In other words, the poly(DMAPS-co-AA) had 1.6 times higher $M_{\mathrm{W}}\left(123823 \mathrm{~g} \mathrm{~mol}^{-1}\right)$ than the poly(AMPS-co-AA) $\left(M_{\mathrm{w}}=77024 \mathrm{~g} \mathrm{~mol}^{-1}\right)$, which could achieve higher steric repulsion between the particles and prevent aggregationinduced sedimentation. To answer this question, we prepared a new poly(AMPS-co-AA) with $M_{\mathrm{w}}$ of $1219631 \mathrm{~g} \mathrm{~mol}^{-1}$, which is $\sim 9.8$ times higher than the $M_{\mathrm{W}}$ of poly(DMAPS-co-AA), grafted it onto the MXene, and observed the long-term colloidal stability in the API brine (Fig. S5†). However, a visible phase separation by the particle sedimentation occurred even earlier, within 30 days, suggesting that the MXene- $g$-poly(AMPS-co-AA) with grafted chains of the highest $M_{\mathrm{w}}$ had the lowest colloidal stability in the API brine. Indeed, the results further support the possible effects of PE-Ca ${ }^{2+}$ interaction on the stability of the PE-grafted MXenes. The poly(AMPS-co-AA) with higher $M_{\mathrm{w}}$, containing more anionic sites, likely underwent more significant complexation with excess $\mathrm{Ca}^{2+}$, dehydration, and even interparticle bridging, all of which could accelerate the particle sedimentation. Overall, the results strongly suggest that the superior colloidal stability of the MXene- $g$-poly(DMAP-co-AA) is due to the anti-polyelectrolyte effect of the grafted PE chains in the API brines containing the divalent $\mathrm{Ca}^{2+}$ ions, not due to any $M_{\mathrm{w}}$ effect.

\subsection{Adsorption properties on mineral substrates}

In addition to securing colloidal stability, it may be equally important to prevent the undesirable adsorption of pollutant scavengers onto porous soil mineral substrates with high specific surface area for high particle mobility in subsurface aquatic environment. We conducted a batch adsorption experiment where either the unmodified pristine MXenes or the PEgrafted MXenes, as an adsorbate, was suspended and agitated for $24 \mathrm{~h}$ in either deionized (DI) water or the API brine in the presence of added alpha-alumina $\left(\alpha-\mathrm{Al}_{2} \mathrm{O}_{3}\right)$ powders, as adsorbent substrates, with an average diameter of $\leq 10 \mu \mathrm{m}$ and a BET surface area of $2.32 \mathrm{~m}^{2} \mathrm{~g}^{-1}$. Fig. 6 shows that the pristine MXenes completely adsorbed onto the mineral substrates in both DI water and API brine, leaving the solution phase visibly clear. In contrast, both PE-grafted MXenes remained in DI water phase (Fig. 6a), not being completely adsorbed onto the mineral substrates, which may be attributed to the (electro)steric 
(a)
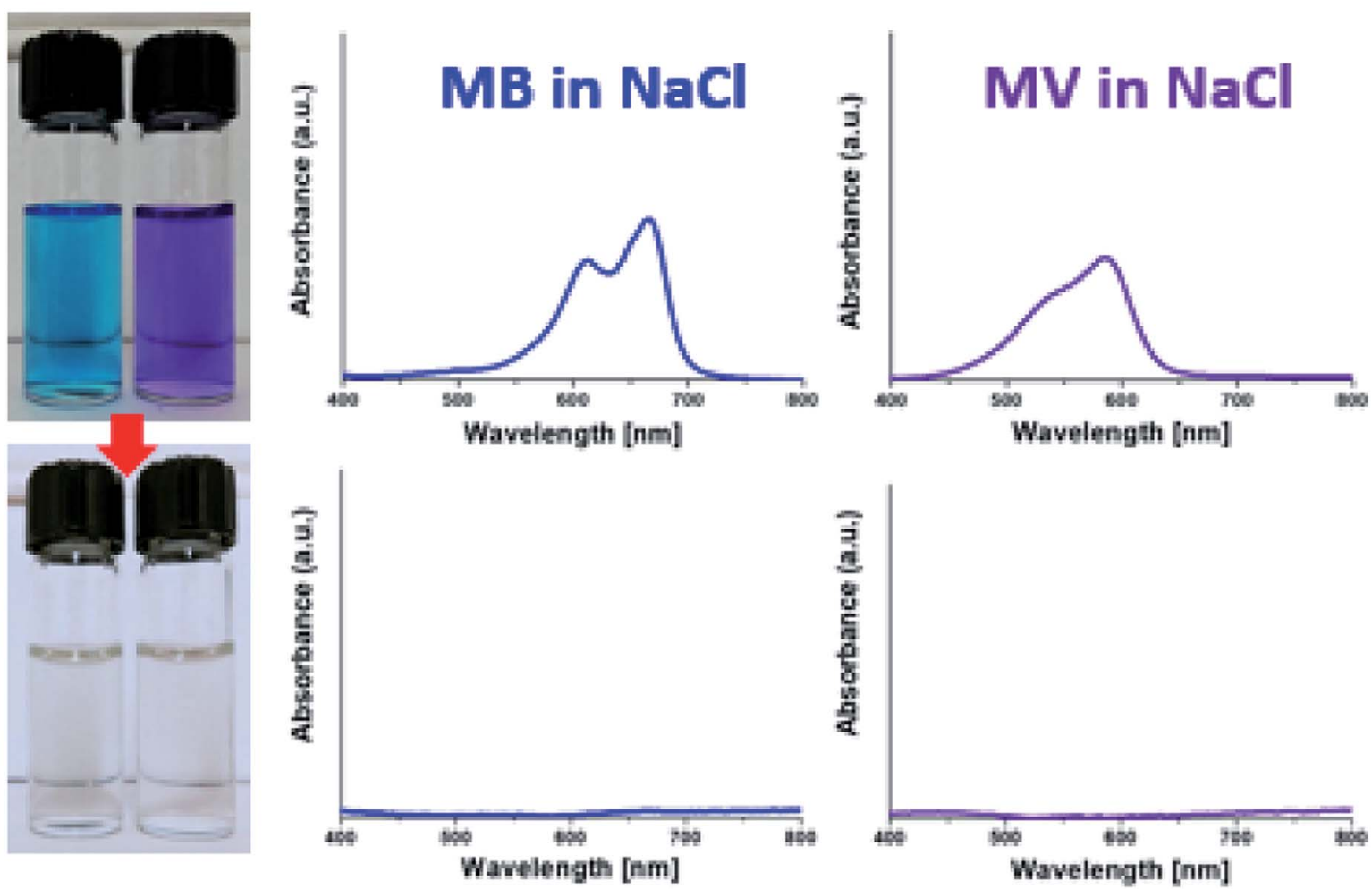

(b)
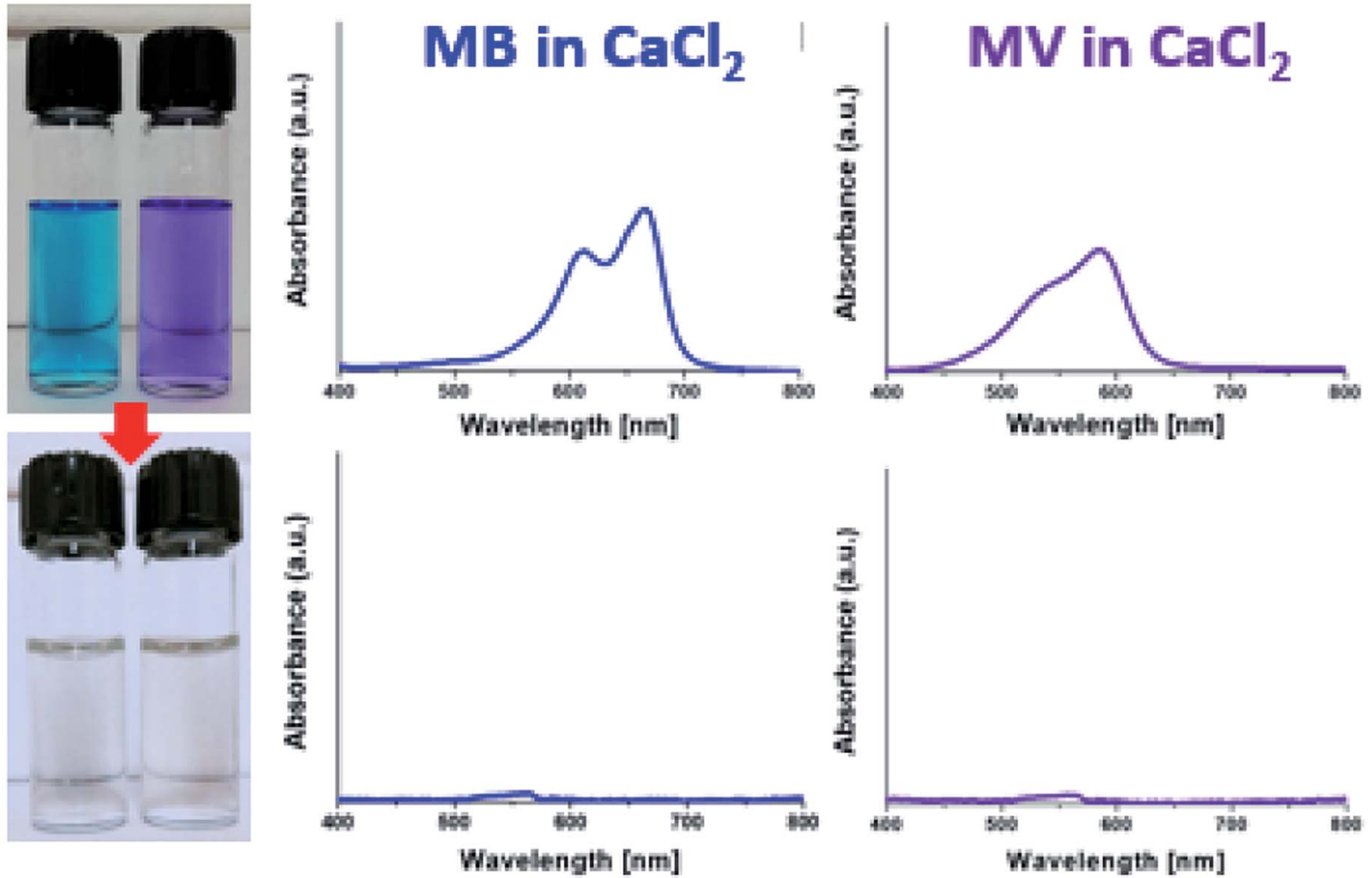

Fig. 8 The removal of $\sim 5$ ppm of methylene blue (MB) or methyl violet (MV) by the MXene-g-poly(DMAPS-co-AA) in (a) $\sim 0.3 \mathrm{M} \mathrm{NaCl}$ and (b) $\sim 0.3 \mathrm{M} \mathrm{CaCl}_{2}$ solutions, demonstrated by photo images and UV-vis spectroscopy.

hindrance by the grafted PEs. ${ }^{7,8,30}$ Interestingly, a stark contrast in the adsorption behavior was observed for the MXenes grafted by anionic and zwitterionic PEs in API brine: whereas the anionic MXene- $g$-poly(AMPS-co-AA), with the grafted PEs of both high (Fig. S6†) and low $M_{\mathrm{w}}$ (Fig. 6b), completely adsorbed onto the mineral substrates, the zwitterionic MXene-g- 
poly(DMAPS-co-AA) showed relatively higher resistance to mineral adsorption (Fig. 6b). The higher resistance to mineral adsorption of the MXene-g-poly(DMAPS-co-AA) in API brine is qualitatively correlated to their higher colloidal stability than that of the MXene- $g$-poly(AMPS-co-AA) in the same environment. The osmotically swollen poly(DMAPS-co-AA) in highsalinity environments likely remained more hydrated on the MXene surface and exerted higher positive excluded volume effects, leaving the adsorption of the PE-grafted MXenes to substrates thermodynamically less favorable. On the other hand, the collapsed poly(AMPS-co-AA) chains were more lyophobic, having the PE-grafted MXenes less resistant to mineral adsorption. Furthermore, the mineral adsorption could possibly be accelerated by $\mathrm{Ca}^{2+}$-mediated bridging between the anionic sites of the grafted PEs and the hydroxyl $\left(-\mathrm{O}^{-}\right)$groups on the $\alpha-\mathrm{Al}_{2} \mathrm{O}_{3}$. We also performed the adsorption experiments for a range of particle concentrations (Fig. 6c) - The maximum adsorption of the MXene- $g$-poly(DMAPS-co-AA) per unit surface area of the $\alpha-\mathrm{Al}_{2} \mathrm{O}_{3}$ in API brine was estimated to be $\sim 0.5 \mathrm{mg}$ $\mathrm{m}^{-2}$ by fitting the experimental data to the Langmuir isotherm model, which is relatively low. ${ }^{30}$ Overall, the MXenes grafted by the zwitterionic PEs displayed superior colloidal stability and resistance to undesirable mineral adsorption in extremesalinity conditions, which are expected to be more effective than the MXenes grafted by the anionic PEs in various subsurface applications. We also attempted to achieve simple physical grafting of the poly(DMAPS-co-AA) on the MXene surface through hydrogen bonding between the PE anchor groups and the MXene surface functionality, avoiding the silane pretreatment and carbodiimide coupling reaction required for chemical (covalent) grafting. However, the product showed poor resistance to the mineral adsorption (Fig. S7 $\dagger$ ), because the weakly adsorbed PEs were likely susceptible to desorption while the particles were vigorously agitated in the presence of mineral substrates with a large specific surface area. This control experiment reveals the importance of achieving robust covalent PE grafting for successful subsurface application of the MXenes.

\subsection{Removal of aqueous contaminants}

It is important that grafting of PE should not completely deactivate the ability of MXenes to adsorb aquatic pollutants. We thus examined the removal efficiency of the PE-grafted MXenes for aqueous pollutants, where aqueous organic dyes were employed for the evaluation. We preliminarily performed a batch experiment where methylene blue (MB), which has been previously employed for studying the adsorption capacity of multi-layered MXenes, ${ }^{15-17}$ was an adsorbate and the unmodified pristine MXene was adsorbent. Based on the Langmuir isotherm analysis, the adsorption capacity of the delaminated MXenes for MB was found to be $121.59 \mathrm{mg} \mathrm{g}^{-1}$ (Fig. 7a), which is higher than $38.85 \mathrm{mg} \mathrm{g}^{-1}$ (ref. 15) and $99.9 \mathrm{mg} \mathrm{g}^{-1}$ (ref. 16) reported for multilayered MXenes in the previous studies. After PE grafting, the adsorption capacity was reduced to $68.04 \mathrm{mg}$ $\mathrm{g}^{-1}$ for the MXene- $g$-poly(AMPS-co-AA) (Fig. 7b) and $67.88 \mathrm{mg}$ $\mathrm{g}^{-1}$ for the MXene-g-poly(DMAPS-co-AA) (Fig. 7c), which is not surprising because many active surface sites of the pristine MXenes for dye adsorption were possibly consumed for condensation of the silane coupling agents. Nonetheless, the PE-grafted MXenes still showed decent adsorption capacities for aqueous MB removal, which are comparable to those of untreated multilayered MXenes. ${ }^{15,16}$

Fig. 8 demonstrates complete removal of $\mathrm{MB}$ or methyl violet (MV), which was dissolved in a $\sim 0.3 \mathrm{M} \mathrm{NaCl}$ or $\mathrm{CaCl}_{2}$ solution at $\sim 5$ ppm, by using MXene- $g$-poly(DMAPS-co-AA). Similar results were demonstrated for the case of using MXene- $g$-poly(AMPS-coAA) in Fig. S8. $\uparrow$ There was no systematic $\mathrm{pH}$ dependence of the dye removal efficiencies by both types of the MXene- $g$-PEs for similarly contaminated solutions with different $\mathrm{pH}$ values in the range of 2-12, as shown in Fig. S9. $\dagger$ We tested the reusability of two types of MXene- $g$-PEs as adsorbents, by washing the adsorbed dyes three times with ethanol and DI water, respectively, and performing the dye removal test under identical conditions. As shown in Fig. S10, $\uparrow$ the dye removal efficiencies decreased by approximately $10 \%$ after the five recycles, but still retained the high values around $90 \%$.

\section{Conclusion}

In this study, we engineered the $\mathrm{Ti}_{3} \mathrm{C}_{2}$-MXene to confer high colloidal stability and low adsorption to mineral substrates in extreme salinity aquatic environments, via the grafting of saltresistant PEs on to MXene surfaces. The MXenes grafted with zwitterionic PEs showed long-term colloidal stability over 6 months in API brine with extreme salinity (ionic strength of $2 \mathrm{M}$ with $\left.182.2 \mathrm{mM} \mathrm{Ca}^{2+}\right)$, and low adsorption $\left(0.5 \mathrm{mg} \mathrm{m}^{-2}\right)$ against $\alpha$-alumina surfaces $\left(2.3 \mathrm{~m}^{2} \mathrm{~g}^{-1}\right)$ in the batch adsorption test. On the other hand, those grafted with well-known sulfonated anionic PEs settled down within a couple of months in API brine and were lost completely during the batch adsorption test conducted under the identical conditions. The superior mobility performances of the MXenes grafted with zwitterionic PEs were attributed to the anti-PE effect of the grafted PE brushes. The PE-grafted MXenes retained satisfactory adsorption capacity of $\sim 68 \mathrm{mg} \mathrm{g}^{-1}$ for methylene blue as a model aqueous organic pollutant, which is comparable to those of conventional nano adsorbents. Overall, the results suggest the great potential of the developed material as a candidate for versatile envrionmental applications ranging from the combined ex situ/in situ remediation, ${ }^{37,57}$ with the MXene- $g$-PEs as a pollutant scavenger, potentially to imaging of subsurface contamination source $z^{2} e^{37}$ in conjunction with the high electronic conductivity of the base material. ${ }^{12}$ Furthermore, the proven colloidal stability in the testing medium (API brine) often employed in the studies on subsurface oil and gas applications strongly suggests new possibilities for using the MXenes, with the grafted PEs, as an additive of nanofluids for down-hole applicsations such as enhanced oil recovery (EOR) and reservoir characterization. ${ }^{30-32,39-42}$

\section{Conflicts of interest}

The authors declare no competing financial interest. 


\section{Acknowledgements}

This research was supported by the 2019 Research and Development Program for "A Study on Surface Modification Techniques of Nanomaterials for in situ Remediation of Contaminated Groundwater and Soil in Gyeonggi-do (19-05-0360-63)" through the Gyeonggi Green Environment Center. This research was supported by Basic Science Research Program through National Research Foundation of Korea (NRF) funded by the Ministry of Education (NRF-2018R1D1A1A02085492). This research was supported by Nano Material Technology Development Program through the National Research Foundation of Korea (NRF) funded by the Ministry of Science, ICT and Future Planning (2009-0082580).

\section{References}

1 R. K. Gautam, M. C. Chattopadhyaya, Advanced Nanomaterials for Wastewater Remediation, CRC Press, Boca Raton, FL, USA 2016.

2 X. M. Ren, J. X. Li, X. L. Tan and X. K. Wang, Comparative Study of Graphene Oxide, Activated Carbon and Carbon Nanotubes as Adsorbents for Copper Decontamination, Dalton Trans., 2013, 42, 5266-5274.

3 J. J. Zhan, T. Zheng, G. Piringer, C. Day, G. L. Mcpherson, Y. F. Lu, K. Papadopoulos and V. T. John, Transport Characteristics of Nanoscale Functional Zerovalent Iron/ Silica Composites for In situ Remediation of Trichloroethylene, Environ. Sci. Technol., 2008, 42, 88718876.

4 Q. Huang, M. Liu, L. Mao, D. Xu, G. Zeng, H. Huang, R. Jiang, F. Deng, X. Zhang and Y. Wei, Surface Functionalized $\mathrm{SiO}_{2}$ Nanoparticles with Cationic Polymers via the Combination of Mussel Inspired Chemistry and Surface Initiated Atom Transfer Radical Polymerization: Characterization and Enhanced Removal of Organic Dye, J. Colloid Interface Sci., 2017, 499, 170-179.

5 S. A. Kim, S. K. Kannan, K. J. Lee, Y. J. Park, P. J. Shea, W. H. Lee, H. M. Kim and B. T. Oh, Removal of $\mathrm{Pb}(\mathrm{II})$ from Aqueous Solution by a Zeolite-Nanoscale Zero-Valent Iron Composite, Chem. Eng. J., 2013, 217, 54-60.

6 D. D. Shao, C. L. Chen and X. K. Wang, Application of Polyaniline and Multiwalled Carbon Nanotube Magnetic Composites for Removal of $\mathrm{Pb}(\mathrm{II})$, Chem. Eng. J., 2012, 185186, 144-150.

7 T. Phenrat, N. Saleh, K. Sirk, R. D. Tilon and G. V. Lowry, Aggregation and Sedimentation of Aqueous Nanoscale Zerovalent Iron Dispersions, Environ. Sci. Technol., 2007, 41, 284-290.

8 N. Saleh, H.-J. Kim, T. Phenrat, K. Matyjaszewski, R. D. Tilton and G. V. Lowry, Ionic Strength and Composition Affect the Mobility of Surface-Modified Fe0 Nanoparticles in WaterSaturated Sand Columns, Environ. Sci. Technol., 2008, 42, 3349-3355.

9 T. Wen, X. L. Wu, X. L. Tan, X. K. Wang and A. W. Wu, OnePot Synthesis of Water-Swellable Mg-Al Layered Double Hydroxides and Graphene Oxide Nanocomposites for
Efficient Removal of As(V) from Aqueous Solutions, ACS Appl. Mater. Interfaces, 2013, 5, 3304-3311.

10 G. X. Zhao, X. B. Huang, X. K. Wang, P. Connor, J. X. Li, S. W. Zhang and J. T. S. Irvine, Synthesis and LithiumStorage Properties of $\mathrm{MnO} /$ Reduced Graphene Oxide Composites Derived from Graphene Oxide Plus the Transformation of $\mathrm{Mn}(\mathrm{VI})$ to $\mathrm{Mn}(\mathrm{II})$ by the Reducing Power of Graphene Oxide, J. Mater. Chem. A, 2015, 3, 297-303.

11 Q. Huang, M. Liu, J. Chen, Q. Wan, J. Tian, L. Huang, R. Jiang, Y. Wen, X. Zhang and Y. Wei, Facile Preparation of $\mathrm{MoS}_{2}$ Based Polymer Composites via Mussel Inspired Chemistry and Their High Efficiency for Removal of Organic Dyes, Appl. Surf. Sci., 2017, 419, 35-44.

12 K. Rasool, R. P. Pandey, P. A. Rasheed, S. Buczek, Y. Gogotsi and K. A. Mahmoud, Water Treatment and Environmental Remediation Applications of Two-Dimensional Metal Carbides (MXenes). Mater, Today, 2019, 30, 80-102.

13 Y. Zhang, L. Wang, N. Zhang and Z. Zhou, Adsorptive Environmental Applications of MXene Nanomaterials: A Review, RSC Adv., 2018, 8, 19895-19905.

14 J. Chen, Q. Huang, H. Huang, L. Mao, M. Liu, X. Zhang and Y. Wei, Recent Progress and Advances in the Environmental Applications of MXene Related Materials, Nanoscale, 2020, 12, 3574-3592.

15 O. Mashtalir, K. M. Cook, V. N. Mochalin, M. Crowe, M. W. Barsoum and Y. Gogotsi, Dye Adsorption and Decomposition on Two-Dimensional Titanium Carbide in Aqueous Media, J. Mater. Chem. A, 2014, 2, 14334-14338.

16 W. Zheng, P. Zhang, W. Tian, X. Qin, Y. Zhang and Z. M. Sun, Alkali treated $\mathrm{Ti}_{3} \mathrm{C}_{2} \mathrm{~T}_{\mathrm{x}}$ MXenes and Their Dye Adsorption Performance, Mater. Chem. Phys., 2018, 206, 270-276.

17 Y. Lei, Y. Cui, Q. Huang, J. Dou, D. Gan, F. Deng, M. Liu, X. Li, X. Zhang and Y. Wei, Facile Preparation of Sulfonic Groups Functionalized MXenes for Efficient Removal of Methylene Blue, Ceram. Int., 2019, 45, 17653-17661.

18 D. Gan, Q. Huang, J. Dou, H. Huang, J. Chen, M. Liu, Y. Wen, Z. Yang, X. Zhang and Y. Wei, Bioinspired Functionalization of MXenes $\left(\mathrm{Ti}_{3} \mathrm{C}_{2} \mathrm{~T}_{\mathrm{x}}\right)$ with Amino Acids for Efficient Removal of Heavy Metal Ions, Appl. Surf. Sci., 2020, 504, 144603.

19 A. Shahzad, K. Rasool, W. Miran, M. Nawaz, J. Jang, K. Mahmoud and D. S. Lee, Two-Dimensional $\mathrm{Ti}_{3} \mathrm{C}_{2} \mathrm{~T}_{\mathrm{x}}$ MXene Nanosheets for Efficient Copper Removal from Water, ACS Sustainable Chem. Eng., 2017, 5, 11481-11488.

20 Y. Ying, Y. Liu, X. Wang, Y. Mao, W. Cao, P. Hu and X. Peng, Two-Dimensional Titanium Carbide for Efficiently Reductive Removal of Highly Toxic Chromium(VI) from Water, ACS Appl. Mater. Interfaces, 2015, 7, 1795-1803.

21 A. Shahzad, K. Rasool, W. Miran, M. Nawaz, J. Jang, K. A. Mahmoud and D. S. Lee, Mercuric Ion Capturing by Recoverable Titanium Carbide Magnetic Nanocomposite, $J$. Hazard. Mater., 2018, 344, 811-818.

22 L. Wang, W. Tao, L. Yuam, Z. Liu, Q. Huang, Z. Cai, J. K. Gibson and W. Shi, Rational Control of the Interlayer Space Inside Two-Dimensional Titanium Carbides for Highly Efficient Uranium Removal and Imprisonment, Chem. Commun., 2017, 53, 12084-12087. 
23 C. E. Ren, K. B. Hatzell, M. Alhabeb, Z. Ling, K. A. Mahmoud and Y. Gogotsi, Charge- and Size-Selective Ion Sieving Through $\mathrm{Ti}_{3} \mathrm{C}_{2} \mathrm{~T}_{\mathrm{x}}$ MXene Membranes, J. Phys. Chem. Lett., 2015, 6, 4026-4031.

24 K. M. Kang, D. W. Kim, C. E. Ren, K. M. Cho, S. J. Kim, J. H. Choi, Y. T. Nam, Y. Gogotsi and H.-T. Jung, Selective Molecular Separation on $\mathrm{Ti}_{3} \mathrm{C}_{2} \mathrm{~T}_{\mathrm{x}}$-Graphene Oxide Membranes during Pressure-Driven Filtration: Comparison with Graphene Oxide and MXenes, ACS Appl. Mater. Interfaces, 2017, 9, 44687-44694.

25 W. Bao, X. Tang, X. Guo, S. Choi, C. Wang, Y. Gogotsi and G. Wang, Porous Cryo-Dried MXene for Efficient Capacitive Deionization, Joule, 2018, 2, 778-787.

26 C. Peng, X. Yang, Y. Li, H. Yu, H. Wang and F. Peng, Hybrids of Two-Dimensional $\mathrm{Ti}_{3} \mathrm{C}_{2}$ and $\mathrm{TiO}_{2}$ Exposing $\{001\}$ Facets toward Enhanced Photocatalytic Activity, ACS Appl. Mater. Interfaces, 2016, 8, 6051-6060.

27 E. Lee, A. VahidMohammadi, B. C. Prorok, Y. S. Yoon, M. Beidaghi and D.-J. Kim, Room Temperature Gas Sensing of Two-Dimensional Titanium Carbide (MXene), ACS Appl. Mater. Interfaces, 2017, 9, 37184-37190.

28 T. Tosco, M. P. Papini, C. C. Viggi and R. Sethi, Nanoscale Zerovalent Iron Particles for Groundwater Remediation: A Review, J. Cleaner Prod., 2014, 77, 10-21.

29 Y. Zou, X. Wang, A. Khan, P. Wang, Y. Liu, A. Alsaedi, T. Hayat and X. Wang, Environmental Remediation and Application of Nanoscale ZeroValent Iron and Its Composites for the Removal of Heavy Metal Ions: A Review, Environ. Sci. Technol., 2016, 50, 7290-7304.

30 H. G. Bagaria, Z. Xue, B. M. Neilson, A. J. Worthen, K. Y. Yoon, S. Nayak, V. Cheng, J. H. Lee, C. W. Bielawski and K. P. Johnston, Iron Oxide Nanoparticles Grafted with Sulfonated Copolymers are Stable in Concentrated Brine at Elevated Temperatures and Weakly Adsorb on Silica, ACS Appl. Mater. Interfaces, 2013, 5, 3329-3339.

31 M. Ranka, P. Brown and T. A. Hatton, Responsive Stabilization of Nanoparticles for Extreme Salinity and High-Temperature Reservoir Applications, ACS Appl. Mater. Interfaces, 2015, 7, 19651-19658.

32 J. Lee, E. Moesari, C. B. Dandamudi, G. Beniah, B. Chang, M. Iqbal, Y. Fei, N. Zhou, C. J. Ellison and K. P. Johnston, Behavior of Spherical Poly(2-acrylamido-2methylpropanesulfonate) Polyelectrolyte Brushes on Silica Nanoparticles up to Extreme Salinity with Weak Divalent Cation Binding at Ambient and High Temperature, Macromolecules, 2017, 50, 7699-7711.

33 Y. Mei, K. Lauterbach, M. Hoffmann, O. V. Borisov, M. Ballauff and A. Jusufi, Collapse of Spherical Polyelectrolyte Brushes in the Presence of Multivalent Counterions, Phys. Rev. Lett., 2006, 97, 158301.

34 B. K. Brettmann, N. Laugel, N. Hoffmann, P. Pincus and M. Tirrell, Bridging Contributions to Polyelectrolyte Brush Collapse in Multivalent Salt Solutions, J. Polym. Sci., Part A: Polym. Chem., 2016, 54, 284-291.

35 Y. Xie, Y. Gao, X. Ren, G. Song, A. Alsaedi, T. Hayat and C. Chen, Colloidal Behaviors of Two-Dimensional Titanium Carbide in Natural Surface Waters: The Role of
Solution Chemistry, Environ. Sci. Technol., 2020, 54, 33533362.

36 C. Power, J. I. Gerhard, M. Karaoulis, P. Tsourlos and A. Giannopoulos, Evaluating Four-Dimensional Time-Lapse Electrical Resistivity Tomography for Monitoring DNAPL Source Zone Remediation, J. Contam. Hydrol., 2014, 162, $27-46$.

37 T. Zhang, G. V. Lowry, N. L. Capiro, J. Chen, W. Chen, Y. Chen, D. D. Dionysiou, D. W. Elliott, S. Ghoshal, T. Hofmann, H. Hsu-Kim, J. Hughes, C. Jiang, G. Jiang, C. Jing, M. Kavanaugh, Q. Li, S. Liu, J. Ma, B. Pan, T. Phenrat, X. Qu, X. Quan, N. Saleh, P. J. Vikesland, Q. Wang, P. Westerhoff, M. S. Wong, T. Xia, B. Xing, B. Yan, L. Zhang, D. Zhou and P. J. J. Alvarez, In situ Remediation of Subsurface Contamination: Opportunities and Challenges for Nanotechnology and Advanced Materials, Environ. Sci.: Nano, 2019, 6, 1283-1302.

38 X. Zhang, Q. Huang, F. Deng, H. Huang, Q. Wan, M. Liu and Y. Wei, Mussel-Inspired Fabrication of Functional Materials and Their Environmental Applications: Progress and Prospects, Appl. Mater. Today, 2017, 7, 222-238.

39 Z. Xue, E. Foster, Y. Wang, S. Nayak, V. Cheng, V. W. Ngo, K. D. Pennell, C. W. Bielawski and K. P. Johnston, Effect of Grafted Copolymer Composition on Iron Oxide Nanoparticle Stability and Transport in Porous Media at High Salinity, Energy Fuels, 2014, 28, 3655-3665.

40 E. E. Urena-Benavides, E. L. Lin, E. L. Foster, Z. Xue, M. R. Ortiz, Y. Fei, E. S. Larsen, A. A. Kmetz, B. A. Lyon, E. Moaseri, C. W. Bielawski, K. D. Pennell, C. J. Ellison and K. P. Johnston, Low Adsorption of Magnetite Nanoparticles with Uniform Polyelectrolyte Coatings in Concentrated Brine on Model Silica and Sandstone, Ind. Eng. Chem. Res., 2016, 55, 1522-1532.

41 H. Park, S. Lim, J. Yang, C. Kwak, J. Kim, J. Kim, S. S. Choi, C. B. Kim and J. Lee, A Systematic Investigation on the Properties of Silica Nanoparticles "Multipoint"-Grafted with Poly(2-acrylamido-2-methylpropanesulfonate-co-acrylic Acid) in Extreme Salinity Brines and Brine-Oil Interfaces, Langmuir, 2020, 36, 3174-3183.

42 J. Lee, G. Beniah, C. B. Dandamudi, J. J. Han, B. A. Lyon, C. A. Norton, N. D. Huffman, L. M. Johnson, J. B. Mecham, G. D. Rothrock, N. Zhou, K. D. Pennell and K. P. Johnston, Noncovalent Grafting of Polyelectrolytes onto Hydrophobic Polymer Colloids with a Swelling Agent, Colloids Surf., A, 2018, 555, 457-464.

43 M. Alhabeb, K. Maleski, B. Anasori, P. Lelyukh, L. Clark, S. Sin and Y. Gogotsi, Guidelines for Synthesis and Processing of Two-Dimensional Titanium Carbide $\left(\mathrm{Ti}_{3} \mathrm{C}_{2} \mathrm{~T}_{\mathrm{x}}\right.$ MXene), Chem. Mater., 2017, 29, 7633-7644.

44 S. Lim, H. Park, J. Yang, C. Kwak and J. Lee, Stable Colloidal Dispersion of Octylated $\mathrm{Ti}_{3} \mathrm{C}_{2}$-MXenes in a Nonpolar Solvent, Colloids Surf., A, 2019, 579, 123648.

45 P. Post, L. Wurlitzer, W. Maus-Friedrichs and A. P. Weber, Characterization and Applications of Nanoparticles Modified in-Flight with Silica or Silica-Organic Coatings, Nanomaterials, 2018, 8, 530. 
46 J. Ederer, P. Janos, P. Ecorchard, J. Tolasz, V. Stengl, H. Benes, M. Perchacz and O. Pop-Georgievski, Determination of Amino Groups on Functionalized Graphene Oxide for Polyurethane Nanomaterials: XPS Quantitation vs. Functional Speciation, $R S C A d v$., 2017, 7, 12464.

47 E. B. Zhulina, T. M. Birshtein and O. V. Borisov, Theory of Ionizable Polymer Brushes, Macromolecules, 1995, 28, 1491-1499.

48 G. Fritz, V. Schadler, N. Willenbacher and N. J. Wagner, Electrosteric Stabilization of Colloidal Dispersions, Langmuir, 2002, 18, 6381-6390.

49 M. Ballauff, Spherical Polyelectrolyte Brush, Prog. Polym. Sci., 2007, 32, 1135-1151.

50 R. Konradi, Interaction of Poly(methacrylic acid) Brushes with Metal Ions: Swelling Properties, Macromolecules, 2005, 38, 4345-4354.

51 A. Ezhova and K. Huber, Contraction and Coagulation of Spherical Polyelectrolyte Brushes in the Presence of $\mathrm{Ag}^{+}$, $\mathrm{Mg}^{2+}$, and $\mathrm{Ca}^{2+}$ Cations, Macromolecules, 2016, 49, 74607468.

52 X. Guo and M. Ballauff, Spherical Polyelectrolyte Brushes: Comparison between Annealed and Quenched Brushes,
Phys. Rev. E: Stat. Phys., Plasmas, Fluids, Relat. Interdiscip. Top., 2001, 64, 051406.

53 N. Su, H. Li, Y. Huang and X. Zhang, Synthesis of Salt Responsive Spherical Polymer Brushes, J. Nanomater., 2015, 2015, 956819.

$54 \mathrm{~J}$. K. Newman and C. L. McCormick, Water-soluble copolymers. 52. sodium-23 NMR studies of ion-binding to anionic polyelectrolytes: poly(sodium 2-acrylamido-2methylpropanesulfonate), poly(sodium 3-acrylamido-3methylbutanoate), poly(sodium acrylate), and poly(sodium galacturonate), Macromolecules, 1994, 27, 5114-5122.

55 C. L. McCormick and D. L. Elliott, Water-Soluble Copolymers. 14. Potentiometric and Turbidimetric Studies of Water-Soluble Copolymers of Acrylamide: Comparison of Carboxylated and Sulfonated Copolymers, Macromolecules, 1986, 19, 542-547.

56 T. Wang, C. Zhao, J. Xu and D. Sun, Enhanced $\mathrm{Ca}^{2+}$ Binding with Sulfonic Acid Type Polymers at Increased Temperature, Colloids Surf., A, 2013, 417, 256-263.

57 H. I. Gomes, C. Dias-Ferreira and A. B. Ribeiro, Overview of In situ and Ex situ Remediation Technologies for PCBContaminated Soils and Sediments and Obstacles for FullScale Application, Sci. Total Environ., 2013, 445-446, 237260. 